\title{
Tratamento cirúrgico da ginecomastia: uma análise criteriosa
}

\author{
Surgical treatment of gynecomastia: a critical analysis
}

\section{Marilho Tadeu Dornelas ${ }^{1}$ \\ Dequitier Carvalho \\ MACHADO $^{2}$ \\ Ana luisa Carneiro Pereira \\ GONÇALVES $^{2}$ \\ Marcília de CÁssia \\ DORNELAS $^{3}$ \\ Marilia de Pádua Dornelas}

Correa $^{4}$

Trabalho realizado na Clínica Plastic Center, Juiz de Fora, MG,

Artigo submetido pelo SGP (Sistema de Gestão de Publicações) da RBCP.

Trabalho apreesntado no III Simpósio do Setor IV do Colégio

Brasileiro de Cirurgiões- II Congresso de Cirurgia da Zona da Mata, realizado na cidade de Juiz de Fora, entre 4 e 6 de dezembro de 2008 .

Artigo recebido: $17 / 6 / 2010$ Artigo aceito: 16/9/2010

\section{RESUMO}

Introdução: A ginecomastia é o aumento no tamanho da glândula mamária masculina, que pode ser fisiológico ou patológico, sendo condição passível de correção. Objetivo: Analisar o tratamento cirúrgico da ginecomastia, apontando suas principais complicações. Método: Duzentos e oitenta e quatro pacientes foram submetidos a cirurgia para correção da ginecomastia entre janeiro de 1998 e setembro de 2008, sendo 94,37\% dos pacientes graus I e II e 5,63\%, grau III. Resultados: Em relação ao total de pacientes, $3,87 \%$ apresentaram seroma e $2,11 \%$, hematoma $(p<0,001)$. A análise dos pacientes graus I e II revela que $0,74 \%$ apresentaram cicatrizes hipertróficas nas incisões de lipoaspiração $(\mathrm{p}<0,001)$. Dentre os pacientes submetidos à técnica de Davidson, $18,75 \%$ apresentaram alargamento do complexo aréolo-mamilar $(\mathrm{p}=0,108)$ e foram submetidos a cirurgia para correção. Conclusão: As técnicas utilizadas para correção de ginecomastia se mostraram eficazes e seguras.

Descritores: Ginecomastia/cirurgia. Mama/cirurgia. Masculino.

\section{SUMMARY}

Introduction: The gynecomastia is an increase in male breast and an important impact on quality of life. However, it is a condition likely to be modified. Objective: To analyze the surgical treatment of gynecomastia pointing the major complications. Methods: Two hundred and eighty-four patients underwent surgery to correct gynecomastia from January 1998 to September 2008, 94.37\% of patients were grade I and II and 5.63\%, grade III. Results: $3.87 \%$ patients had seromas and $2.11 \%$, hematoma $(\mathrm{p}<0.001)$. Patients grade I and II had keloids in incision of liposuction $(0.74 \%)$ and patients grade III presented areolar enlargement. Conclusion: The techniques used for treatment of gynecomastia were considered safe and effective with low complication rate.

Descriptors: Gynecomastia/surgery. Breast/surgery. Male.

1. Membro Titular da Sociedade Brasileira de Cirurgia Plástica (SBCP); Mestre em Saúde pela Universidade Federal de Juiz de Fora.

2. Acadêmico da Faculdade de Medicina da Universidade Federal de Juiz de Fora.

3. Membro Especialista da Sociedade Brasileira de Cirurgia Plástica.

4. Membro Titular da SBCP, Chefe do Serviço de Cirurgia Plástica do Hospital Universitário da Universidade Federal de Juiz de Fora. 


\section{INTRODUÇÃO}

O termo ginecomastia designa um aumento no tamanho da glândula mamária masculina, que pode ser fisiológico ou patológico ${ }^{1}$. Esta é a patologia de mama mais frequente nos homens ${ }^{2}$, estando presente em cerca de $38 \%$ dos pacientes jovens ${ }^{3}$. Clinicamente, trata-se de uma massa firme e elástica, situada atrás e ao redor do complexo aréolo-mamilar (CAM). Histologicamente, caracteriza-se pela proliferação de tecido adiposo, fibroso e ductal, em proporções variáveis ${ }^{1}$.

O aumento da mama em homens deve sempre ser considerado patológico, exceto nos períodos neonatal, puberal e senil, nos quais a ginecomastia pode ser fisiológica ${ }^{1,2}$. A patogênese da ginecomastia está relacionada à alteração no equilíbrio entre andrógenos e estrogênios ${ }^{1}$. Esta se deve à diminuição na produção de andrógenos e aumento na produção de estrogênio, ao aumento na transformação dos precursores de andrógenos em estrogênio, a alterações nos receptores de estrogênios ou ao aumento da sensibilidade da mama a este hormônio. Porém, na maioria dos pacientes, a causa destas alterações hormonais não é identificada ${ }^{1,2}$.

A maioria dos casos de ginecomastia patológica requer abordagem cirúrgica, cuja técnica difere de acordo com a gravidade. O tratamento clínico é recomendado durante a fase de proliferação do tecido glandular, sendo realizada a abordagem cirúrgica em caso de falha ${ }^{3}$.

As principais indicações cirúrgicas são a reconstrução do contorno masculino do tórax, o diagnóstico de lesões nodulares suspeitas ${ }^{4} \mathrm{e}$ a presença de ginecomastia dolorosa ${ }^{3}$. As deformidades estéticas causadas pela ginecomastia acarretam importante impacto psicológico e social nos pacientes, comprometendo a qualidade de vida, o que, na maioria das vezes, leva o paciente a procurar tratamento.

O objetivo deste estudo é analisar o tratamento cirúrgico da ginecomastia, apontando suas principais complicações.

\section{MÉTODO}

Foram incluídos neste estudo 284 pacientes submetidos a procedimento cirúrgico para tratamento de ginecomastia, na clínica Plastic Center, no período entre janeiro de 1998 e setembro de 2008.

O diagnóstico de ginecomastia foi realizado por meio de avaliação clínica, com enfoque na idade, presença e duração de sinais e sintomas de doenças correlacionadas, como insuficiência renal, insuficiência hepática, hipertireoidismo, hipogonadismo e síndrome de Klinefelter, entre outras; consumo de drogas, como álcool, anfetaminas, maconha e opioides; exposição a agentes estrogênicos.

Para a escolha da técnica cirúrgica mais adequada, os pacientes foram classificados de acordo com a gravidade.
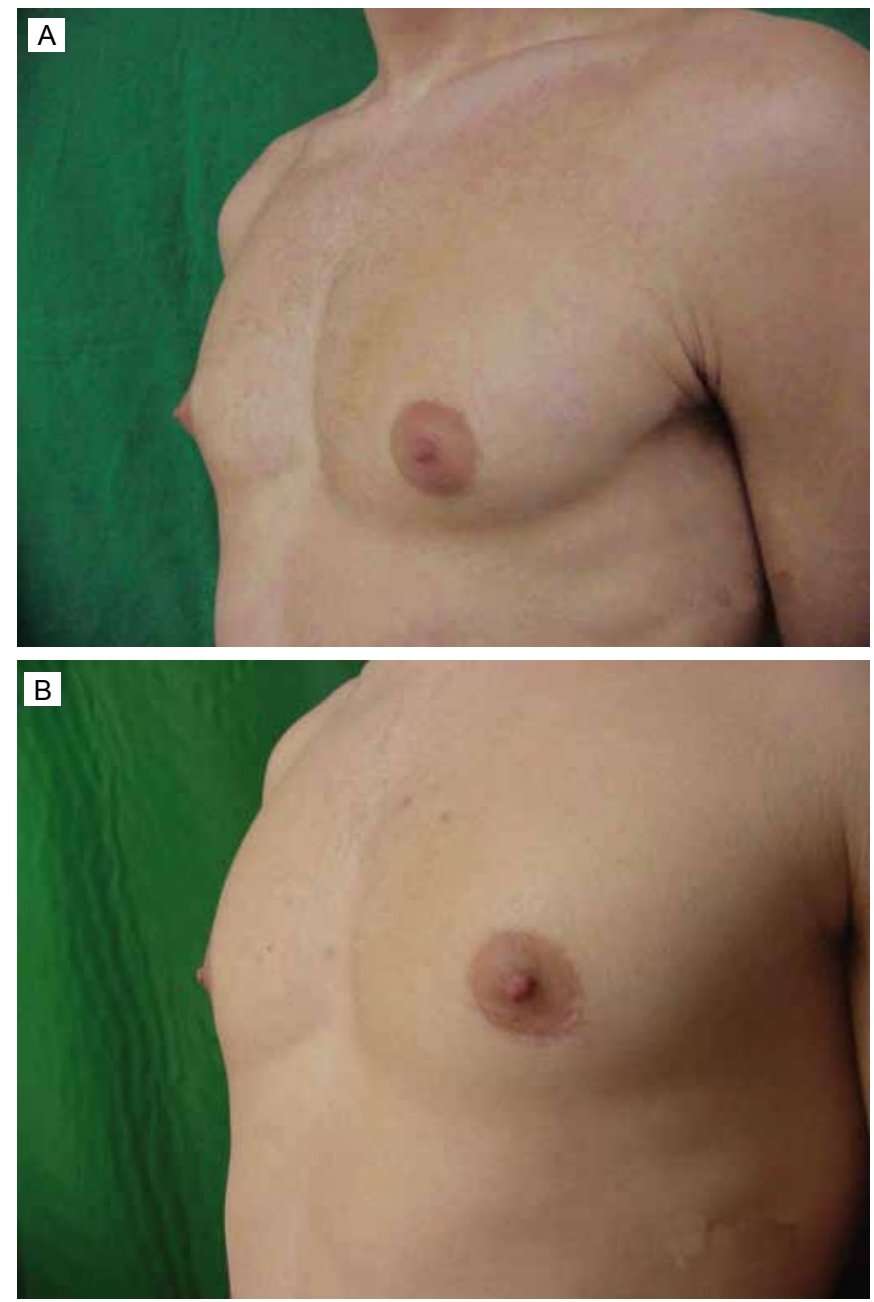

Figura 1 - Ginecomastia grau I; A: Vista no pré-operatório e B: No pós-operatório tardio, com cicatriz pouco perceptivel e redução no abaulamento do CAM.

Considerou-se grau I os pacientes que apresentavam crescimento do diâmetro e protrusão glandular restrito à região areolar; grau II, aqueles que apresentavam hipertrofia de todos os componentes estruturais da mama, com ou sem excesso de pele; e grau III, aqueles com características similares ao grau II, porém apresentando ptose.

Nos pacientes graus I (Figura 1) e II (Figura 2), foi realizada a ressecção do tecido glandular, deixando um pequeno coxim sob a aréola, e lipoaspiração nos casos com excesso de tecido adiposo. Devido à semelhança na técnica, os pacientes graus I e II foram avaliados em conjunto.

Nos pacientes grau III (Figura 3), foi realizada a técnica de Davidson ${ }^{5}$, que se baseia em lipoaspiração do tecido adiposo redundante, desepitelização da metade superior da mama que será responsável pela nutrição do CAM e ressecção da pele e tecido glandular presente na porção inferior da mama. 

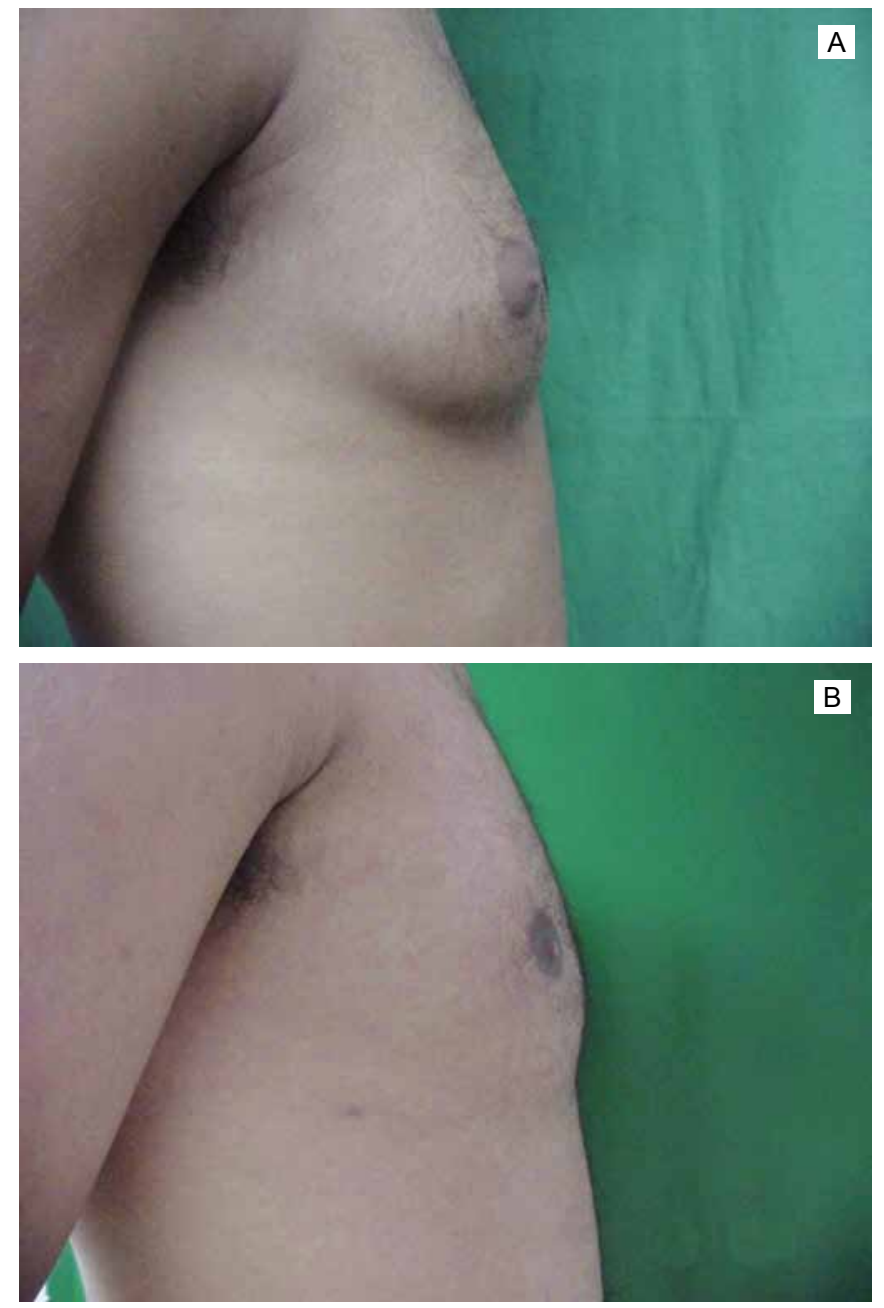

Figura 2 - Ginecomastia grau II, A: Vista no préoperatório e B: No pós-operatório tardio.

No pós-operatório, os pacientes foram orientados a utilizar malha compressiva durante um mês. As atividades físicas foram suspensas por quatro semanas após a cirurgia. O acompanhamento dos pacientes foi de um ano.

Os resultados foram considerados insatisfatórios na presença de seroma, hematoma, cicatrizes hipertróficas ou alargamento do CAM, pois estas são as complicações mais relevantes nas técnicas estudadas ${ }^{2,6}$.

A análise estatística foi realizada através do teste quiquadrado.

\section{RESULTADOS}

Dentre os pacientes analisados, apenas 5,63\% apresentaram ginecomastia grau III, isso se deve à maior relação desta com alterações hormonais importantes. Observou-se, também,
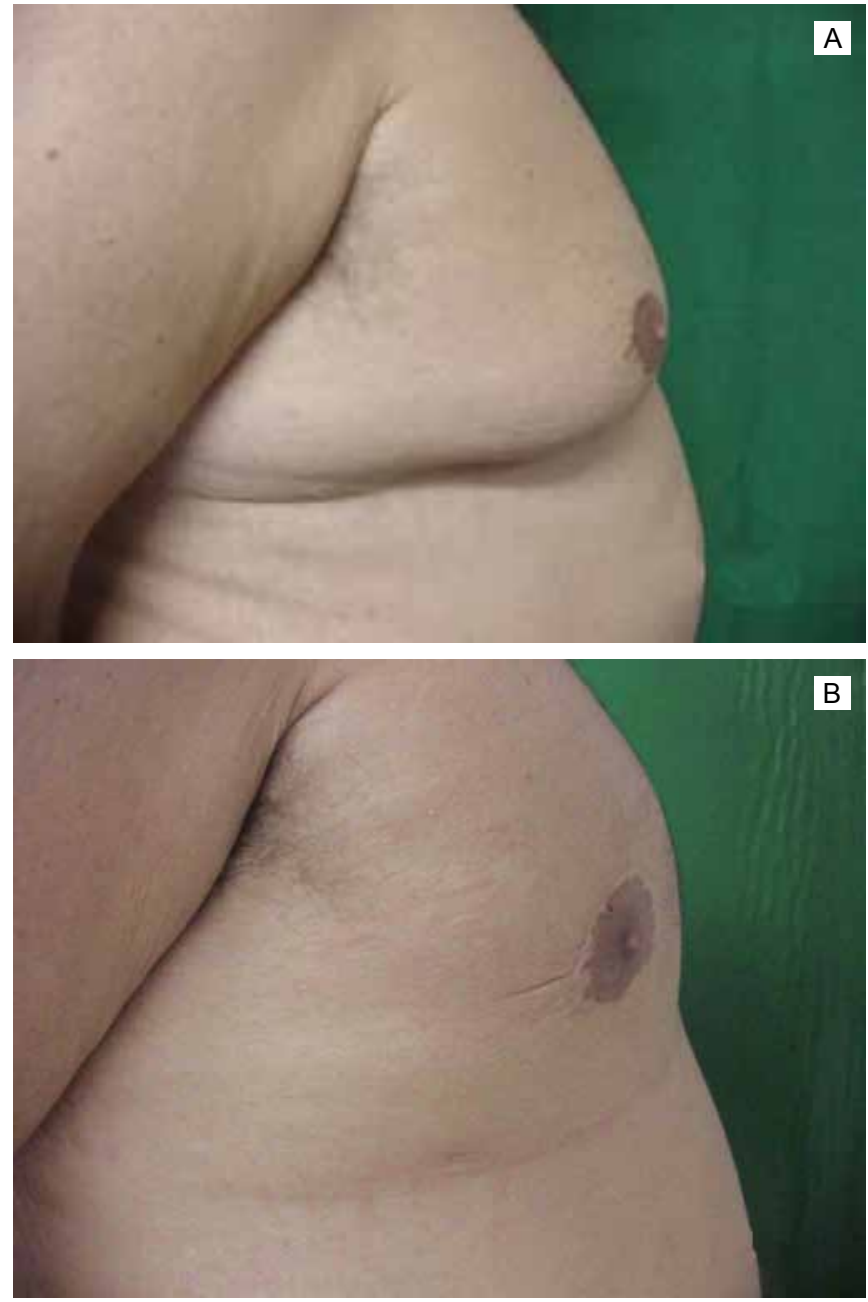

Figura 3 - Ginecomastia grau III, A: Vista no préoperatório e B: No pós-operatório tardio.

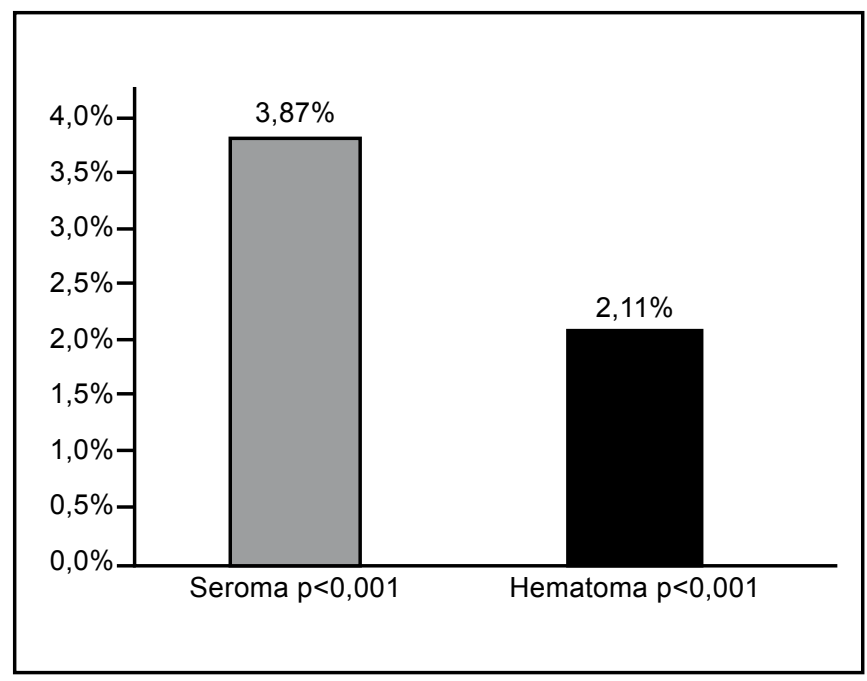

Figura 4 - Incidência de complicações no total de pacientes. 
que 5,28\% dos pacientes apresentavam ginecomastia unilateral, índice inferior ao apresentado pela literatura ${ }^{2,6}$.

Aincidência de seromas e hematomas no total de pacientes encontra-se na Figura 4. Estas complicações foram tratadas satisfatoriamente de forma conservadora.

A análise dos pacientes graus I e II revela que $0,74 \%$ apresentaram cicatrizes hipertróficas nas incisões de lipoaspiração $(\mathrm{p}<0,001)$. Dentre os pacientes submetidos à técnica de Davidson, 18,75\% apresentaram alargamento do complexo aréolo-mamilar $(\mathrm{p}=0,108)$ e foram submetidos à cirurgia para correção. Não ocorreram casos de necrose, infecções ou recidiva da ginecomastia.

\section{DISCUSSÃO}

Ginecomastia causa grande impacto emocional e social nos homens, principalmente durante o período da puberdade $^{7}$. As alterações na forma e no tamanho da mama levam os pacientes a interromper atividades cotidianas e de lazer, acarretando redução na qualidade de vida. A maioria dos pacientes requer tratamento devido a este impacto e às alterações psicológicas decorrentes ${ }^{1}$.

O tratamento cirúrgico da ginecomastia apresenta grandes variações na literatura, o que reflete a ausência de protocolos amplamente aceitos. Atualmente, tem-se focado mais no tratamento por lipoaspiração devido ao melhor resultado estético e à menor incidência de complicações, quando comparado à excisão glandular. No entanto, a escolha da técnica cirúrgica deve ser baseada em características individuais, como grau de hipertrofia glandular.

A análise adequada do material retirado deve ser realizada, independentemente da técnica aplicada, para identificação de possíveis lesões malignas ${ }^{6}$. No presente estudo, não foram encontrados achados de malignidade.
A incidência de hematoma e seroma foi inferior à apresentada na literatura.

\section{CONCLUSÃO}

Os principais objetivos do tratamento cirúrgico da ginecomastia são: redução do abaulamento na região peitoral, eliminação da prega inframamária, posicionamento correto do CAM, remoção do excesso de pele, simetrização do tórax e aréolas, deixando cicatrizes pequenas ${ }^{8}$. Esses objetivos foram atingidos de forma satisfatória nos pacientes analisados, demonstrando a eficácia e a segurança das técnicas utilizadas.

\section{REFERÊNCIAS}

1. Celebioğlu S, Ertaş NM, Ozdil K, Oktem F. Gynecomastia treatment with subareolar glandular pedicle. Aesthetic Plast Surg. 2004;28(5):281-7.

2. Oroz J, Pelay MJ, Roldán P. Ginecomastia: tratamiento quirúrgico. Anales Sis San Navarra. 2005;28(2):109-16.

3. Fruhstorfer BH, Malata CM. A systematic approach to the surgical treatment of gynecomastia. Br J Plast Surg. 2003;56(3):237-46.

4. Colombo-Benkmann M, Buse B, Stern J, Herfarth C. Indications for and results of surgical therapy for male gynecomastia. Am J Surg. 1999;178(1):60-3.

5. Dornelas MT, Teixeira VC, Yung CM, Andrade IA, Corrêa MPD, Gonçalves CM. Tratamento cirúrgico da ginecomastia grau III. Rev Bras Cir. 1996;86(5):255-60.

6. Handschin AE, Bietry D, Husler R, Banic A, Constantinescu M. Surgical management of gynecomastia: a 10-year analysis. World J Surg. 2008;32(1):38-44

7. Gheita A. Gynecomastia: the horizontal ellipse method for its correction. Aesthetic Plast Surg. 2008;32(5):795-801.

8. Cordova A, Moschella F. Algorithm for clinical evaluation and surgical treatment of gynaecomastia. J Plast Reconstr Aesthet Surg. 2008;61(1):41-9 